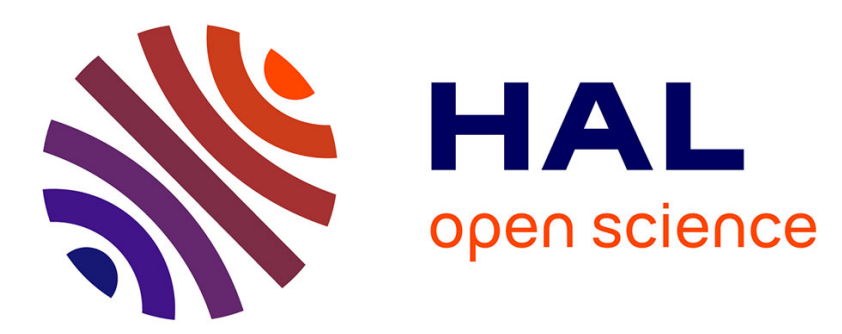

\title{
Degenerate scale for the Laplace problem in the half-plane; Approximate logarithmic capacity for two distant boundaries
}

\author{
Alain Corfdir, Guy Bonnet
}

\section{- To cite this version:}

Alain Corfdir, Guy Bonnet. Degenerate scale for the Laplace problem in the half-plane; Approximate logarithmic capacity for two distant boundaries. Engineering Analysis with Boundary Elements, 2013, 37 (5), pp.219-224. 10.1016/j.enganabound.2013.02.009 . hal-00806771

\section{HAL Id: hal-00806771}

\section{https://hal.science/hal-00806771}

Submitted on 17 Jan 2016

HAL is a multi-disciplinary open access archive for the deposit and dissemination of scientific research documents, whether they are published or not. The documents may come from teaching and research institutions in France or abroad, or from public or private research centers.
L'archive ouverte pluridisciplinaire HAL, est destinée au dépôt et à la diffusion de documents scientifiques de niveau recherche, publiés ou non, émanant des établissements d'enseignement et de recherche français ou étrangers, des laboratoires publics ou privés. 


\title{
Degenerate scale for the Laplace problem in the half-plane; Approximate logarithmic capacity for two distant boundaries
}

\author{
A. Corfdir ${ }^{\mathrm{a}, *}, \mathrm{G}$. Bonnet ${ }^{\mathrm{b}}$ \\ a Université Paris-Est, Laboratoire Navier (UMR 8205), CNRS, ENPC, IFSTTAR, F-77455 Marne-la-Vallée, France \\ 'Université Paris Est, Laboratoire Modélisation et Simulation Multi-Echelle, MSME UMR 8208 CNRS, 5 boulevard Descartes, 77454 Marme la Vallée Cedex, France
}

\section{A B S T R A C T}

We study the problem of finding a degenerate scale for Laplace equation in a half-plane. It is shown that if the boundary condition on the line bounding the half-plane is of Dirichlet type, there is no degenerate scale. In the case of a boundary condition of Neumann type, there is a degenerate scale, which is shown to be the same as the one for the symmetrized contour with respect to the boundary line in the full plane. We show next a formula for obtaining the degenerate scale of a domain made of two parts, when the components are far from each other, which allows to obtain the degenerate scale for the symmetrized contour. Finally, we give some examples of evaluation of the degenerate scale both by an approximate formula and by a numeric evaluation using integral methods. These evaluations show that the approximate solution is still valid for small values of the distance between symmetrized contours.

Keywords:

Laplace equation

Boundary element method

Plane problems

Green's function

Degenerate scale

Half-plane

Exterior problem

\section{Introduction}

One considers for a given contour $S$ all contours obtained by any scaling of $S$. Early results by Jaswon [21], based on the work of Muskhelishvili [23], showed that for any boundary $S$ of a plane domain, there is one and only one degenerate scale which leads to the non-invertibility of the integral operator: $q \mapsto \psi_{q}(x)=$ $-\int_{S} \ln \|x-y\| q(y) d S_{y}$. More precisely, it can be shown that there is a distribution $q(x)$ such that the application $\psi_{q}(x)$ is null for any $x$. Hayes and Kellner [17] have shown using complex variables that a degenerate scale is reached when the "transfinite diameter" of the outer domain, defined from a conformal mapping of the domain from the outer unit circle, is equal to 1. From another point of view, the transfinite diameter defined in the complex plane is equal to the logarithmic capacity as shown by Hille [19]. The logarithmic capacity, whose definition is recalled in Section 5 , is defined without using a conformal mapping, which makes it easier to handle. Yan and Sloan [29] give a review of the main properties of the logarithmic capacity. It is possible to derive analytically the logarithmic capacity of some domains by using conformal mapping, for example for regular N-gon domains [22]. The numerical calculation of the logarithmic capacity has been studied by Dijkstra and Hochstenbach [16] and Chen et al. [5].

\footnotetext{
Corresponding author. Tel.: + 33164153521

E-mail addresses: corfdir@cermes.enpc.fr (A. Corfdir).
} Guy.Bonnet@univ-mlv.fr (G. Bonnet).
The degenerate scale problem is still investigated by different authors: Yan and Sloan [29] have studied the case of an open boundary, Coscia and Russo [14] have studied the case of a Lipschitz boundary. The problem has been extended to multiply connected domains [6,8]. Many authors have discussed how to eliminate this problem by scaling [12], by adding a constant to the fundamental solution [12,5], by adding an unknown and an additional equation $[11,20]$, by adding an additional collocation point [4] or by using an hypersingular formulation as noted by Chen et al. [5]. Practically, a 2D problem can be very often considered as an approximation of a 3D problem at the vicinity of a finite line source. In this case, a specific kernel depending of the geometry of the 3D problem can be defined [1]. This kernel ensures that the scale of the $2 \mathrm{D}$ problem is smaller than the degenerate scale if a condition ensuring that 2D modeling is well adapted to the original 3D problem is satisfied. A more extended review on the problem of degenerate scale for Laplace equation can be found in Chen [3]. More generally, some authors have studied the degenerate scale for other equations having a logarithmic term in the Green function: elasticity in the plane $[18,13,26,27,9,10]$, biharmonic equation in the plane $[15,6]$. These equations exhibit similar though more complicated behaviors, with generally several degenerate scales.

This paper is devoted to the study of degenerate scales for problems in a half-plane, which seems, from our knowledge, not having been studied with as much extent as the problem in a full plane. Elementary solutions of the Laplace equation for the halfplane is based on the method of images. This method has been 
found very early by Thomson [25] and is still the object of research (see e.g. $[8,7]$ ). In this paper, we use the results established for the plane and draw next basic results for the case of the half-plane, either for Dirichlet or Neumann boundary conditions at the boundary of the half-plane. An approximate of the degenerate scale for the exterior problem is given, when the diameter of the inner boundary is small compared with the distance between this inner boundary and the boundary line of the half-plane.

\section{Relation between the degenerate scale in a half-plane and an associated problem in the full plane}

We first recall the link between the loss of unicity of the integral boundary equation and the existence of a non-trivial solution of the equation

$\int_{S} G(x-y) q(y) \mathrm{d} S_{y}=0, \quad x \in S$

where $G(x, y)=-\ln \|x-y\| / 2 \pi$. Assuming the loss of unicity for Dirichlet condition, making the difference between two solutions, we get a function $u(x)$ with $q(x)=\partial u / \partial n$ satisfying the following boundary equation, $\frac{1}{2} u(x)+\int_{S}(\partial G / \partial n)(x-y) u(y) \mathrm{d} S_{y}=\int_{S} G(x-$ $y) q(y) \mathrm{d} S_{y}$ with $u(x)=0$ for $x \in S$ and $q$ being a non-null function on $S$. The boundary integral equation then gives $\int_{S} G(x-$ $y) q(y) \mathrm{d} S_{y}=0$ for $x \in S$. Conversely, if there exists $q$ a non-trivial solution of (1), then the function $u(x)=\int_{S} G(x-y) q(y) \mathrm{d} S_{y}$ is a nonnull solution of the Laplace equation which vanishes on $S$.

The above argument for the plane can be applied for the halfplane using the appropriate kernel. Then, the degenerate scale in the half-plane is related to the non-invertibility of the operator $q \mapsto \psi_{q}(x)=-\int_{S} G_{\alpha}(x-y) q(y) \mathrm{d} S_{y}$ where $G_{\alpha}$ is the Green's function associated with conditions applied to the boundary line of the half-plane. We can consider two different problems in the halfplane according to the type of the condition at the line $A$ bounding the half-plane: Neumann condition if the normal derivative is null on $\Delta$ or Dirichlet conditions if the function is null on $\Delta$ (Fig. 1 ).

We consider the standard Green solution for Laplace equation in the plane: $G(x, y)=-\ln \|x-y\| / 2 \pi$. For the half-plane, the image method gives the following Green function:

$G_{\alpha}(x, y)=G(x, y)+\epsilon G(\bar{x}, y)$

where $\bar{x}$ is the image of $x, \alpha=N$ and $\epsilon=1$ for Neumann condition and $\alpha=D$ and $\epsilon=-1$ for Dirichlet condition on line 4 . Considering that many results are found in the literature on the degenerate scale in the plane, we intend to build a relation between the degenerate scale in the half-plane for a given boundary and the degenerate scale for an associate problem in the full plane.

Theorem 1. If a problem in the half-plane is at a degenerate scale, then an associate problem can be built, which is at a degenerate scale in the full plane.

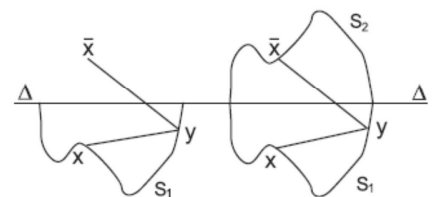

Fig. 1. The image method (left) and the symmetrization of the boundary (right).
Proof. We extend the function $q$ defined on $S_{1}$ to a function $q$ defined on $S_{1} \cup S_{2}$ in the following way:

$q_{\alpha}(y)= \begin{cases}q(y) & \text { if } y \in S_{1} \\ \epsilon q(\bar{y}) & \text { if } \bar{y} \in S_{2}\end{cases}$

with $\alpha=N$ and $\epsilon=1$ for Neumann condition and $\alpha=D$ and $\epsilon=-1$ for Dirichlet condition on line 4 . Using $G(\bar{x}, y)=G(x, \bar{y})$, we can write

$$
\begin{aligned}
\int_{S_{1}} G_{\alpha}(x, y) q(y) \mathrm{d} S_{y} & =\int_{S_{1}}(G(x, y)+\epsilon G(\bar{x}, y)) q(y) \mathrm{d} S_{y} \\
& =\int_{S_{1}} G(x, y) q(y) \mathrm{d} S_{y}+\int_{S_{1}} G(x, \bar{y}) \epsilon q(y) \mathrm{d} S_{y} \\
& =\int_{S_{1}} G(x, y) q(y) \mathrm{d} S_{y}+\int_{S_{2}} G(x, y) q_{\alpha}(y) \mathrm{d} S_{y} \\
& =\int_{S_{1} \cup S_{2}} G(x, y) q_{\alpha}(y) \mathrm{d} S_{y}
\end{aligned}
$$

If the domain $S_{1}$ is at a degenerate scale, the integral operator is not invertible for the problem in the half-plane with Neumann or Dirichlet condition on the 4 line; therefore, there is a non-nul function $q$ such that: $\int_{S_{1}} G_{\alpha}(x, y) q(y) \mathrm{d} S_{y}=0$.

Then, the extended function $q_{\alpha}$ is such (4) that $\int_{S_{1} \cup S_{2}} G(x, y) q_{\alpha}(y) \mathrm{d} S_{y}=0$.

Hence $S_{1} \cup S_{2}$ is at the degenerate scale for the problem in the plane. This proves Theorem 1 .

This result is particularly useful, because it allows to apply all results obtained in the plane for the symmetrized domain $S_{1} \cup S_{2}$.

Before to use this associate domain in Section 5, two general results on the degenerate problems associated with Dirichlet or Neumann boundary conditions will be established.

\section{The Laplace problem in the half-plane with a Dirichlet condition on the boundary line}

In this section, we assume that a null Dirichlet condition is applied on the boundary line. In this case, we establish the following theorem:

Theorem 2. There is no degenerate scale for the Laplace problem in the half-plane with Dirichlet condition on the boundary line.

Proof. Assumebycontradictionthattheboundary $S_{1}$ isatthedegeneratescalefortheLaplaceproblemin thehalf-planewithDirichletconditiononthelineboundingthehalf-plane. Thereexistsanon-nullfunctionqsuchthat: $\int_{S_{1}} G_{D}(x, y) q(y) \mathrm{d} S_{y}=0$.

If we change the scale of the problem by a factor $\lambda$ (Fig. 2), we define

$q_{\lambda}(y)=q\left(\frac{y}{\lambda}\right)$

From the definition of $G_{D}(x, y)$, we get $G_{D}(\lambda x, \lambda y)=G_{D}(x, y)$. Hence, we can write

$\int_{\lambda S_{1}} G_{D}(x, y) q_{\lambda}(y) \mathrm{d} S_{y}=\lambda \int_{S_{1}} G_{D}(\lambda x, \lambda y) q(y) \mathrm{d} S_{y}$

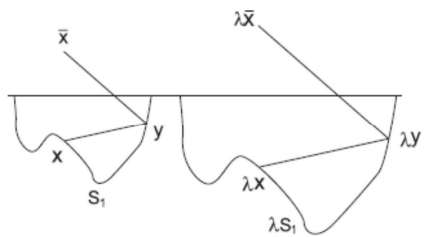

Fig. 2. Change of scale. 


$$
=\lambda \int_{S_{1}} G_{D}(x, y) q(y) \mathrm{d} S_{y}=0
$$

From this equation and from the conclusion of the preceding section, we conclude that the symmetrized problem on the boundary $S_{1} \cup S_{2}$ in the plane would have an infinity of degenerate scales. There is a contradiction with the known fact that this degenerate scale is unique. This proves finally Theorem 2 .

This result shows that the search of the solution of the boundary problem in a domain within the half-plane associated with a null Dirichlet condition on the boundary line can be effected without worrying about a possible degenerate scale.

\section{The Laplace problem in the half-plane with Neumann condition on the boundary line}

Now, the half-plane is studied when applying a Neumann condition on the boundary line. It is shown in this case that the situation is analogous with the full plane:

Theorem 3. There is one and only one degenerate scale for the Laplace problem in the half-plane with Neumann condition on the boundary line.

Proof. We consider a boundary which is symmetric with respect to a line 4 ; its two symmetric components are $S_{1}$ and $S_{2}$. If the scale is degenerate, there is a non-null function such that

$\int_{S_{1} \cup S_{2}} G(x, y) q(y) d S_{y}=0$

The above equation is true for all $x$, so we can write: $\int_{S_{1} \cup S_{2}} G(\bar{x}, y) q(y) \mathrm{d} S_{y}=0$. As $G(\bar{x}, y)=G(x, \bar{y})$, we have: $\int_{S_{1} \cup S_{2}} G(\bar{x}, y)$ $q(y) \mathrm{d} S_{y}=\int_{S_{1} \cup S_{2}} G(x, \bar{y}) q(y) \mathrm{d} S_{y}=\int_{S_{1} \cup S_{2}} G(x, y) q(\bar{y}) \mathrm{d} S_{y}$. So, we get

$\int_{S_{1} \cup S_{2}} G(x, y) q(\bar{y}) d S_{y}=0$

Turning $y$ into $\bar{y}$ in Eqs. (6)-(7), we get

$\int_{S_{1} \cup S_{2}} G(x, \bar{y}) q(\bar{y}) \mathrm{d} S_{y}=0$

$\int_{S_{1} \cup S_{2}} G(x, \bar{y}) q(y) \mathrm{d} S_{y}=0$

Adding Eqs. (7), $\epsilon$ (8), (9), $\epsilon$ (10), with $\epsilon= \pm 1$ we get

$\int_{S_{1} \cup S_{2}}(G(x, y)+\epsilon G(x, \bar{y}))(q(y)+\epsilon q(\bar{y})) d S_{y}=0$

We define

$q_{s}=q(y)+q(\bar{y})$

$q_{a}=q(y)-q(\bar{y})$

Rewriting (11) for $\epsilon=1$ and -1 , we have

$\int_{S_{1} \cup S_{2}} G_{N}(x, y) q_{s}(y) \mathrm{d} S_{y}=0$

$\int_{S_{1} \cup S_{2}} G_{D}(x, y) q_{a}(y) \mathrm{d} S_{y}=0$

As $G_{N}(x, y) q_{s}(y)=G_{N}(x, \bar{y}) q_{s}(\bar{y})$ and $G_{D}(x, y) q_{a}(y)=G_{D}(x, \bar{y}) q_{a}(\bar{y})$, we can now write

$\int_{S_{1}} G_{N}(x, y) q_{s}(y) \mathrm{d} S_{y}=0$

$\int_{S_{1}} G_{D}(x, y) q_{a}(y) \mathrm{d} S_{y}=0$
From (16) we deduce that $q_{a}=0$ as there is no degenerate scale for the Laplace problem in the half-plane with Dirichlet condition on the bounding line. Then $q(y)=q(\bar{y})$, and as $q(y)$ is a non-null function $q_{s}(y)$ is also a non-null function, and $S_{1}$ is at the degenerate scale. This proves finally Theorem 3 , which shows that the problem in the halfplane with a condition of Neumann's type on the boundary line is completely similar to the problem in the full plane, leading to the need to evaluate the degenerate scale. $\square$

The following section is therefore devoted to an evaluation of the degenerate scale in the case of Neumann's boundary condition.

\section{Approximate of the logarithmic capacity for a domain with} two connected components

It has been shown in Section 2 that the problem of the degenerate scale for the half-plane with Neumann condition on the bounding line reduces to the problem of the degenerate scale of the symmetrized domain in the plane. We will now pay attention to a particular class of domains made of two components as in Fig. 3(left) and we will also consider a slightly more general problem of two components far from each other and not necessarily symmetric as in Fig. 3(right). Each component $S_{i}$ has a circumscribed circle of radius $r_{i}$, the distance between the centers of the two circumscribed circles is denoted by $d$. Some authors have investigated the related problem of two equal cylindrical conductors with equal charge and have given rather complicated solutions using elliptic functions or series $[28,2]$. In this section, we focus on simple approximate of the logarithmic capacity in the case of two distant components; each component is a simple curve and not necessary a circle.

The logarithmic capacity is equal to 1 for the degenerate scale. We recall the definition of the logarithmic capacity $C_{S_{1} \cup S_{2}}$ of $S_{1} \cup S_{2}$

$-\ln \left(C_{S_{1} \cup S_{2}}\right)=\inf \int_{S_{1} \cup S_{2}} \int_{S_{1} \cup S_{2}} \ln \frac{1}{\|x-y\|} q(x) q(y) \mathrm{d} S_{x} \mathrm{~d} S_{y}$

with $q \geq 0$ and $\int_{S_{1} \cup S_{2}} q(x) \mathrm{d} S_{x}=1$. The integral in (17) can be split into four parts

$\int_{S_{1} \cup S_{2}} \int_{S_{1} \cup S_{2}} \ln \frac{1}{\|x-y\|} q(x) q(y) \mathrm{d} S_{x} \mathrm{~d} S_{y}=\int_{S_{1}} \int_{S_{1}} \ln \frac{1}{\|x-y\|} q(x) q(y) \mathrm{d} S_{x} \mathrm{~d} S_{y}$ $+\int_{S_{1}} \int_{S_{2}} \ln \frac{1}{\|x-y\|} q(x) q(y) \mathrm{d} S_{x} \mathrm{~d} S_{y}+\int_{S_{2}} \int_{S_{1}} \ln \frac{1}{\|x-y\|} q(x) q(y) \mathrm{d} S_{x} \mathrm{~d} S_{y}$ $+\int_{S_{2}} \int_{S_{2}} \ln \frac{1}{\|x-y\|} q(x) q(y) \mathrm{d} S_{x} \mathrm{~d} S_{y}$

We consider the second integral $\int_{S_{1}} \int_{S_{2}} \ln (1 /\|x-y\|) q(x) q(y) \mathrm{d} S_{x} \mathrm{~d} S_{y}$
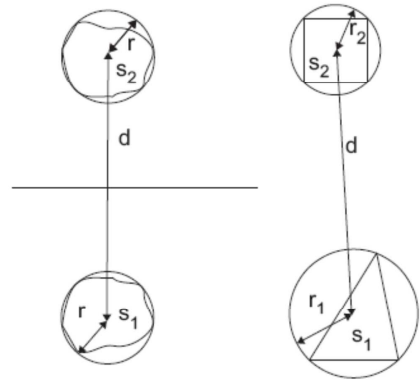

ig. 3. Degenerate scale for two components when $d \gg r$ : symmetric case (left) general case (right). 


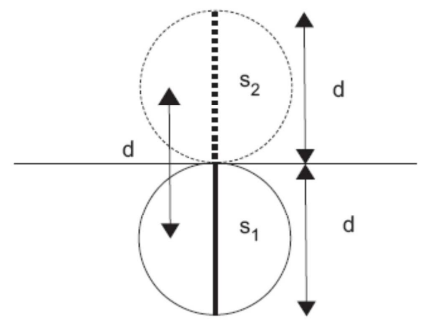

Fig. 4. The case of a segment.

in (18). If $x \in S_{2}$ and $y \in S_{1}$, hence $(d-2 r)<\|x-y\|<(d+2 r)$ with $r=\sup \left(r_{1}, r_{2}\right)$. So we have the following inequalities:

$\int_{S_{1}} \int_{S_{2}} \ln \frac{1}{(d+2 r)} q(x) q(y) \mathrm{d} S_{x} \mathrm{~d} S_{y}$

$\leq \int_{S_{1}} \int_{S_{2}} \ln \frac{1}{\|x-y\|} q(x) q(y) \mathrm{d} S_{x} \mathrm{~d} S_{y}$

$\leq \int_{S_{1}} \int_{S_{2}} \ln \frac{1}{(d-2 r)} q(x) q(y) \mathrm{d} S_{x} \mathrm{~d} S_{y}$

Denoting by $Q_{1}=\int_{S_{1}} q(y) \mathrm{d} S_{y}$ and $Q_{2}=\int_{S_{2}} q(x) \mathrm{d} S_{x}=1-Q_{1}$, we deduce from (19)

$\ln \frac{1}{(d+2 r)} Q_{1} Q_{2} \leq \int_{S_{1}} \int_{S_{2}} \ln \frac{1}{\|x-y\|} q(x) q(y) \mathrm{d} S_{x} \mathrm{~d} S_{y} \leq \ln \frac{1}{(d-2 r)} Q_{1} Q_{2}$

From Eq. (18) and from the right inequality of (20), we deduce the following inequality:

$\int_{S_{1} \cup S_{2}} \int_{S_{1} \cup S_{2}} \ln \frac{1}{\|x-y\|} q(x) q(y) \mathrm{d} S_{x} \mathrm{~d} S_{y} \leq \int_{S_{1}} \int_{S_{1}} \ln \frac{1}{\|x-y\|} q(x) q(y) \mathrm{d} S_{x} \mathrm{~d} S_{y}$

$+2 Q_{1} Q_{2} \ln \frac{1}{(d-2 r)}+\int_{S_{2}} \int_{S_{2}} \ln \frac{1}{\|x-y\|} q(x) q(y) \mathrm{d} S_{x} \mathrm{~d} S_{y}$

We note that for $i=1,2$

$$
\inf _{\substack{q \geq 0 \\ \int_{S_{i}} q(x) \mathrm{d} S_{x}=Q_{i}}} \int_{S_{i}} \int_{S_{i}} \ln \frac{1}{\|x-y\|} q(x) q(y) \mathrm{d} S_{x} \mathrm{~d} S_{y}=-Q_{i}^{2} \ln C_{S_{i}}
$$

Taking the inf of the two parts of the inequality (21) and using (22), we get

$-\ln \left(C_{S_{1} \cup S_{2}}\right) \leq \inf _{Q_{1}+Q_{2}=1}\left(-Q_{1}^{2} \ln C_{S_{1}}+2 Q_{1} Q_{2} \ln \frac{1}{(d-2 r)}-Q_{2}^{2} \ln C_{S_{2}}\right)$

Substituting $1-Q_{1}$ for $Q_{2}$, the right part of the above inequality is a quadratic polynomial of $Q_{1}$ and is extremal for

$Q_{1}=\frac{\ln (d-2 r)-\ln \left(C_{S_{2}}\right)}{2 \ln (d-2 r)-\ln \left(C_{S_{1}}\right)-\ln \left(C_{S_{2}}\right)}$

and the extremum is

$A=-\frac{\ln ^{2}(d-2 r)-\ln \left(C_{S_{1}}\right) \ln \left(C_{S_{2}}\right)}{2 \ln (d-2 r)-\ln \left(C_{S_{1}}\right)-\ln \left(C_{S_{2}}\right)}$

The radius of the circumscribed circle satisfies $r \geq C_{S_{i}}$ for $i=1,2$ [29]. It can be checked for $d \gg r \geq C_{S_{i}}$ that $A$ is a minimum and that the corresponding value of $\left.Q_{1} \in\right] 0,1[$.

As $d \gg r$, we have: $\ln (d-r)=\ln (d)+O(r / d)$. We deduce

$A=-\frac{1}{2} \frac{\ln ^{2}(d)-\ln \left(C_{S_{1}}\right) \ln \left(C_{S_{2}}\right)}{\ln (d)-\ln \sqrt{C_{S_{1}} C_{S_{2}}}}+O(r / d)$
This leads to

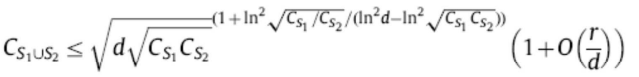

Using also the left inequality of (20) in the same way, we finally conclude

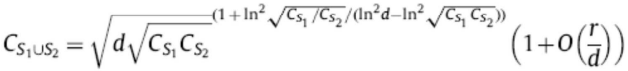

If $C_{S_{1}}$ and $C_{S_{1}}$ are equal, we get the simpler formula

$C_{S_{1} \cup S_{2}}=\sqrt{d C_{S_{1}}}+O\left(\frac{r}{\sqrt{d}}\right)$

These relations have been established under the condition $d \gg r$. This simple closed form of the logarithmic capacity of $S_{1} \cup$ $S_{2}$ will be compared with other values of this capacity, obtained either in closed form or numerically, for configurations where distance between $S_{1}$ and $S_{2}$ will be not necessarily very large.

\section{Comparison of the approximate value of the logarithmic} capacity with closed forms and numerical evaluations

\subsection{Case of a domain having the shape of a segment}

We test our approximate of the logarithmic capacity for two cases which do not satisfy the condition $d \gg r$. We consider first a segment of d length $S_{1}, C_{S_{1}}$ denotes its logarithmic capacity (Fig. 4).

We consider the symmetric segment $S_{2}$. The new segment $S_{1} \cup$ $S_{2}$ has its logarithmic capacity twice larger than $S_{1}$. As the logarithmic capacity is scale proportional, we have: $C_{S_{1} \cup S_{2}}=2 C_{S_{1}}$. The approximate gives: $C_{S_{1} \cup S_{2}} \approx \sqrt{d C_{S_{1}}}$. Eliminating $C_{S_{1} \cup S_{2}}$ from these two relations we get

$C_{S_{1}} \approx \mathrm{d} / 4$

In that case, the approximate turns out to be the exact value, even if both domains are not apart from each other.

\subsection{Case of a rectangle}

We consider the rectangle of $S_{1}$ whose sides are $d, \sqrt{2} d$ (Fig. 5). We consider also the symmetric rectangle $S_{2}$. The new rectangle logarithmic capacity $C_{S_{1} \cup S_{2}}$ is equal to $\sqrt{2} C_{S_{1}}$. The approximate formula gives: $C_{S_{1} \cup S_{2}} \approx \sqrt{d C_{S_{1}}}$. Eliminating $C_{S_{1} \cup S_{2}}$ from these two relations we get

$C_{S_{1}} \approx 0.5 d$

This can be compared with a numeric evaluation using boundary elements (see Appendix A) which gives a not too far value: $0.7102 d$.

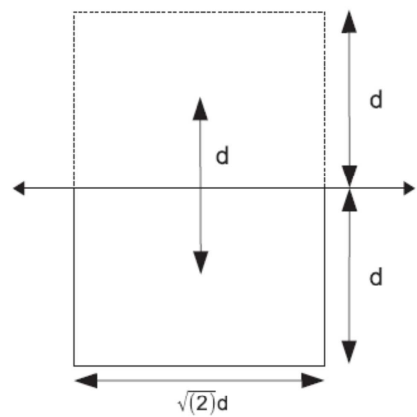

Fig. 5. The case of a rectangle with sides $d, \sqrt{2} d$ and its symmetrized. 


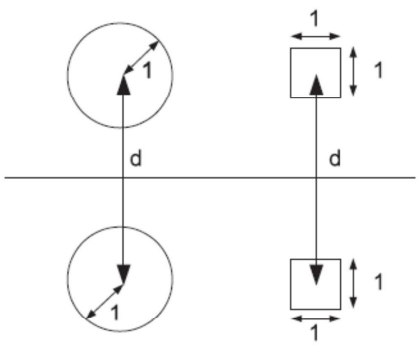

Fig. 6. The cases of two disjoint circles of radius one and of two disjoint squares of side one.

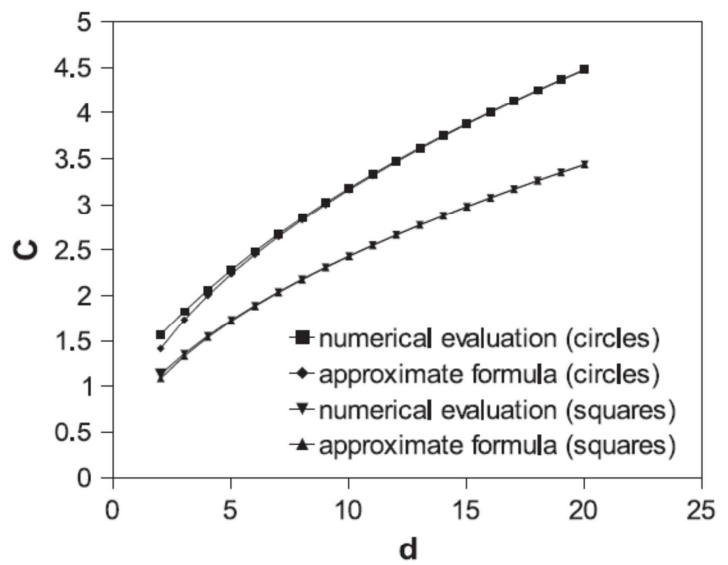

Fig. 7. Comparison of numerical evaluation and of approximate formula.

\subsection{Case of two disjoint circles and squares}

We consider two equal circles of radius one, the length between their centers being denoted by $d$ (Fig. 6). For a circle, the logarithmic capacity is equal to its radius and the approximate formula reduces to $\sqrt{d}$ for circles of radius one. Similarly, for two distant squares of side one, the approximate formula reduces to $\sqrt{0.59017 d}$ as the logarithmic capacity of a square of side one is $\approx 0.59017$ [24].

Fig. 7 shows comparison between the numerical evaluation of the logarithmic capacity by the method of Appendix A and the approximate formula for both cases, squares and circles. The results are displayed only for $d<20$, because for higher values, numerical results are identical with the approximate solution. However, it can be seen that the approximate formula produces satisfying results even when both domains are at very close positions, similarly with the results obtained in both previous applications.

\section{Conclusion}

We have considered the degenerate scale for the Laplace problem in the half-plane with Dirichlet or Neumann boundary condition on the straight boundary line. Using symmetry properties, we have shown that for the first case, there is no degenerate scale and that for the second case the degenerate scale is the same as the one obtained for the symmetrized problem in the plane. We have established an asymptotic formula for the logarithmic capacity of a set of two distant components from the logarithmic capacity of the original domain and from the distance between the components. In addition, it was shown that the asymptotic formula is very efficient even if a domain and its symmetrized are not too far apart. This turns out to be an efficient way for obtaining a satisfying evaluation of the logarithmic capacity of the symmetrized domain, leading to the degenerate scale in the half-plane with Neumann condition on the boundary line.

\section{Appendix A. Numerical computation of the logarithmic capacity by boundary elements}

One considers a domain whose boundary is $S$. The discretization of $S$ in boundary elements leads to the matrix $[G]$ containing the elements $G_{i j}$ defined by

$$
G_{i j}=\int_{S} N_{j} \frac{1}{2 \pi} \ln \left(\frac{a}{\left\|x_{i}-y\right\|}\right) \mathrm{d} S y
$$

where $x_{i}$ are collocation points on $S$ and $y$ is a current integration point on $S, N_{j}$ being the interpolation function related to node $x_{j}$.

The logarithmic capacity is found by looking for the value of $a$ producing a matrix $[G]$ which is singular. The studied examples have shown that the value of $a$ is estimated with an approximation around $10^{-4}$ for a discretization of the contour made of 400 points. This method is simple to program when $[G]$ is already evaluated some authors have suggested more elaborate methods [16,5]. A more computationally economical option would be to search for the generalized eigenvalue of $\left(G_{0}, B\right)$ where $G_{0}=G(a=1)$ et $B_{i j}=C \otimes D$ with $C=(1,1,1, \ldots)$ and $D_{j}=1 / 2 \pi \int_{S} N_{j} \mathrm{~d} S_{y}$.

\section{References}

[1] Bonnet G, Corfdir A, Nguyen M-T. On the solution of exterior plane problems by the boundary element method; a physical point of view, submitted for publication

[2] Cade R. The integral equation solution of two dimensional electrostatic problems involving circular cylinders. J Inst Maths Appl 1980;25:211-30.

[3] Chen J-T. Dual BEM since 1986. In: 2006 forum on advanced engineering computation. National Center for Research on Earthquake Engineering. Taiwan; 2006

[4] Chen J-T, Lee C-F, Chen I-L, Lin J-H. An alternative method for degenerate scale problems in boundary element methods for two dimensional Laplace equation. Int J Numer Methods Eng 2002;26(1):559-69.

[5] Chen J-T, Lin S-R, Chen K-H. Degenerate scale problem when solving Laplace equation by BEM and its treatment. Int J Numer Methods Eng 2005;62(2):233-6

[6] Chen J-T, Shen W-C. Degenerate scale for multiply connected Laplace problems. Mech Res Commun 2006;34:69-77.

[7] Chen J-T, Shieh H-C, Lee Y-T, Lee J-W. Bipolar coordinates, image method and the method of fundamental solutions for Green functions of Laplace problems containing circular boundaries. Eng Anal Boundary Elem 2011:35:236-43.

[8] Chen J-T, Shieh H-C, Tsai J-J, Lee J-W. A study on the method of fundament solutions using an image concept. Appl Math Model 2010;34:4253-66.

[9] Chen Y-Z, Lin X-Y. Regularity condition and numerical examination for degenerate scale problem of BIE for exterior problem of plane elasticity. Eng Anal Boundary Elem 2008:32:811-23.

[10] Chen Y-Z, Lin X-Y, Wang Z-X. Evaluation of the degenerate scale for BIE in plane elasticity and antiplane elasticity by using conformal mapping. Eng Anal Boundary Elem 2009;33:147-58.

[11] Christiansen S. Integral equations without a unique solution can be made useful for solving some plane harmonic problems. J Inst Maths App

[12] Christiansen S. On two methods for elimination of non-unique solutions of an integral equation with logarithmic kernel. Appl Anal 1982;13:1-18.

[13] Constanda C. On non-unique solutions of weakly singular integral equations in plane elasticity. QJ Mech Appl Math 1994:47(2):261-8.

14] Coscia V, Russo R. Some remarks on the Dirichlet problem in plane exterior domains. Ric Math 2007:56:31-41.

[15] Costabel M, Dauge M. Invertibility of the biharmonic single layer potential operator. Integr Equat Oper Theory 1996;24:46-67.

[16] Dijkstra W. Hochstenbach M. Numerical approximation of the logarithmic capacity. 〈http://www.win.tue.nl/ hochsten/pdf/logcap.pdf $\rangle ; 2009,18$.

[17] Hayes J. Kellner $R$. The eigenvalue problem of a pair of coupled integral equations arising in the numerical solution of Laplace equation. SIAM J Appl Math 1972;22(3):503-13.
[18] Heise U. The spectra of some integral operators for plane elastostatical boundary value problem. J Elasticity 1978:8(1):47-79.

20] Hsiao G. On the stability of integral equations of the first kind with logarithmic kernels. SIAM Rev 1986;4(2):179-82.

[21] Jaswon MA. Integral equation methods in potential theory. Proc. R. Soc. London Ser. A Math. Phys. Sci. 1963;275(360):23-32

[22] Kuo S-R, Chen J-T, Lee J-W, Chen Y-W. Analytical derivation and numerical Kuo S-R, Chen J-T, Lee J-W, Chen Y-W. Analytical derivation and numerical
experiments of degenerate scale for regular $\mathrm{N}$-gon domains in BEM. Appl Math Comput 2013:219:5668-83.

[23] Muskhelishvili N. Singular integral equations: boundary problems of function theory and their application to mathematical physics. Noordhoof, Groningen; 1953
[24] Pólya G, Szzegô G. Isoperimetric inequalities in mathematical physics. Princeton University Press; 1951.

25] Thomson W. Electric images, extract from a letter to Mr Liouville, London: MacMillan; 1845.

[26] Vodička R, Mantič V. On invertibility of elastic-single layer potential operator. J Elasticity 2004:74:147-73.

[27] Vodička R, Mantič V. On solvability of a boundary integral equation of the first kind for Dirichlet boundary value problem in plane elasticity. Comput Mech 2008:41:817-26.

[28] Whipple FJW. Equal parallel cylindrical conductors in electrical problems. Proc R Soc London A 1920;96:465-74.

[29] Yan Y Sloan I On integral equations of the first kind with logarithmic kernels. J Integr Equations Appl 1988;1:549-79. 